\title{
Highly Sensitive Detection of Organophosphate Insecticides Using Biosensors Based on Genetically Engineered Acetylcholinesterase and Poly(3,4-Ethylenedioxythiophene)
}

\author{
Tomasz Sikora, ${ }^{1}$ Georges Istamboulie, ${ }^{2}$ Elena Jubete, ${ }^{1}$ Estibalitz Ochoteco, ${ }^{1}$ \\ Jean-Louis Marty, ${ }^{2}$ and Thierry Noguer ${ }^{2}$ \\ ${ }^{1}$ New Materials Department, CIDETEC-Centre for Electrochemical Technologies, Parque Tecnológico de San Sebastián, \\ Paseo Miramón 196, 20009 Donostia-San Sebastian, Spain \\ ${ }^{2}$ Université de Perpignan Via Domitia, IMAGES, Batiment S, 52 Avenue Paul Alduy, 66860 Perpignan Cedex, France
}

Correspondence should be addressed to Thierry Noguer, noguer@univ-perp.fr

Received 14 September 2010; Accepted 24 January 2011

Academic Editor: Jiri Homola

Copyright (C) 2011 Tomasz Sikora et al. This is an open access article distributed under the Creative Commons Attribution License, which permits unrestricted use, distribution, and reproduction in any medium, provided the original work is properly cited.

A poly(3,4-ethylenedioxythiophene) (PEDOT) conducting ink is presented as a new electroactive material to be incorporated in acetylcholinesterase-(AChE-) based screen printed biosensors, acting not only as a conducting template but also as an electrochemical mediator for thiocholine oxidation. Two different strategies have been studied for the chemical synthesis of PEDOT: (a) a classical oxidative polymerisation and (b) a more innovative enzymatic polymerisation, giving a water-soluble PEDOT. The use of this water-soluble conducting polymer as mediator in screen-printed biosensors enables its deposition by printing like the rest of the layers. Highly sensitive acetylcholinesterase-(AChE-) based screen-printed biosensors have been constructed using both classical and enzymatic PEDOT, in combination with genetically modified AChE. These electrodes allow the measurement of thiocholine oxidation at potentials of $100 \mathrm{mV}$ versus $\mathrm{Ag} / \mathrm{AgCl}$ reference electrode through the mediation of PEDOT. Inhibition of thiocholine production in presence of CPO allow for detection of this pesticide in concentrations as low as $1 \cdot 10^{-10} \mathrm{M}$.

\section{Introduction}

Biosensors are increasingly becoming powerful tools in clinical diagnostics, drug detection, and food and environmental monitoring. This is the reason behind the great amount of research focused on new materials and immobilisation strategies in these electroanalytical devices. In the last years, some of these studies have been focused on the development of low cost screen-printed biosensors, which are well suited for mass production and portable devices [1]. As a means to obtain low-cost and disposable biosensors most of screen-printed designs are based on successive layers of graphite or carbon paste including a polymeric binder [25]. The incorporation of an electrochemical mediator and an appropriately immobilized enzyme enables the construction of biosensors suitable for the selective detection of a specific compound.
Numerous studies of screen-printed electrode construction have been published, where the electrode surface modification has been performed using different mediators, with the main goal of decreasing the working potential and thus avoiding electrochemical interferences. As an example, electrochemical oxidation of thiocholine has been achieved at lower potentials using a graphite sensors modified using an appropriate redox mediator like phtalocyanine salts [610] or 7,7,8,8-tetracyanoquinodimethane (TCNQ) [11-15] can be used as chemical mediator. These two mediators incorporated in the graphite paste allow the electrochemical oxidation of thiocholine at $100 \mathrm{mV}$ versus $\mathrm{Ag} / \mathrm{AgCl}$ reference electrode.

On the other hand, the field of intrinsically conducting polymers (ICPs) has attracted great attention due to their interesting electrical and optical properties. Since their discovery in 1977, these materials have been investigated 
for countless technological applications, such as organic lightweight batteries, microelectronics, optical displays, antistatic coatings, and electromagnetic shielding $[16,17]$. They have also been used as components for biosensors. Some groups succeeded in the entrapment of biomolecules in an electrogenerated conducting polymeric film improving the analytical performance of the conventional immobilisation techniques [18-20]. However, the use of an electropolymerised conducting polymer implies an additional stage in the manufacturing process and, therefore, a more expensive mass production.

Apart from being electrochemically generated, conducting polymers can also be chemically synthesised. These highly promising materials have traditionally been synthesised by monomer oxidation in the presence of a strong oxidant, and they usually show high insolubility and intractability, once synthesised. Hence, due to their insolubility in common solvents, the preparation of aqueous dispersions of ICPs by a chemical oxidative polymerisation is a classical way to tackle this problem using colloidal dispersions instead of real solutions [21]. Beyond this conventional synthesis method, the use of enzymes as biocatalysts for the synthesis of conducting polymers is being studied in the very recent last years as an innovative "green-synthesis-process" alternative [22-28]. Enzymes can offer environmentally benign reaction conditions [29], high yield of polymerisation, and higher control in regioregularity and stereochemistry [30, 31 ], consequently resulting in highly soluble and processable conducting polymers [32]. The first enzymatically polymerised poly(3,4-ethylenedioxythiophene) (PEDOT) was reported in 2007 [27], the polymerisation was performed in presence of polystyrene sulfonate and using horseradish peroxidase as catalyst. The resulting PEDOT showed excellent film formation ability, being promising for multiple applications.

In our previous work, a PEDOT conducting ink was presented as a new electrode material to be incorporated in acetylcholinesterase-(AChE-) based screen-printed biosensors [33]. The PEDOT was obtained by a chemical oxidative polymerisation, and it was observed to act not only as a conducting template but also as an electrochemical mediator for thiocholine oxidation, having the advantage that it did not need to be applied by electro polymerization but could directly be incorporated as a last layer during the screen-printing process. The present paper supposes a step forward for the PEDOT-mediated detection of thiocholine/pesticides. In this case, the film formation ability of PEDOT obtained by the innovative enzymatic polymerisation was employed to obtain a new type of PEDOTmediated screen-printed biosensors. Their electrochemical behaviour towards thiocholine oxidation was compared with that one for the electrodes obtained using classical PEDOT and cobalt phtalocyanine, which is established as a common mediator for this system. Both the electrochemically and enzymatically synthesised PEDOT are proved in this work to constitute new promising electrochemical mediators to be used during the fabrication process of screen-printed biosensors without the need of additional expensive steps such as electropolymerisation.

\section{Experimental}

2.1. Chemicals and Stock Solutions. Ethylenedioxythiophene (EDOT) monomer (99\%), poly(sodium 4-styrenesulfonate) (PSS), Horseradish Peroxidase (HRP, EC 1.11.1.7, type II, 150-200 units/mg solid), and ammonium peroxydisulphate (APS) were purchased from Sigma-Aldrich Chemicals S.A. Hydrogen peroxide $\left(\mathrm{H}_{2} \mathrm{O}_{2}, 30 \mathrm{wt} \%\right)$ and hydrochloric acid $(\mathrm{HCl}, 1 \mathrm{~N})$ were obtained from Quimibacter S.L. The concentration of HRP stock solution was $3 \mathrm{mg} / \mathrm{mL}$. Acetylcholinesterase (AChE) B394 mutation was provided by Protein Bio Sensor (PBS, Toulouse, France). Before immobilisation, enzymatic activities were measured spectrophotometrically using 5, 5' -dithiobis(2-nitrobenzoic acid) (DTNBEllman's reagent) provided by Sigma. The substrate acetylthiocholine chloride (ATCh) was purchased from Sigma. A 0.1 M ATCh stock solution was daily prepared in water and stored at $4^{\circ} \mathrm{C}$.

The organophosphorus insecticide chlorpyrifos-oxon (phosphoric acid, O,O-diethyl O-(3,5,6-trichloro-2-pyridinyl) ester) (CPO) was purchased from Dr Ehrenstorfer (Augsburg, Germany). Stock solution of pesticide $\left(10^{-3} \mathrm{M}\right)$ was prepared in acetonitrile and stored at $4^{\circ} \mathrm{C}$.

Polyvinyl alcohol functionalised with methyl pyridinium methyl sulfate (PVA-SbQ) was provided by Toyo Gosei Kogyo Co (Chiba, Japan).

The pastes used for screen-printing, Electrodag PE-410, 423SS, 452SS, and 6037SS, were obtained from Acheson (Plymouth, UK), cobalt-phtalocyanine-modified carbon paste (CoPC) was purchased from Gwent Electronic Materials, Ltd (Gwent, UK). Transparent PVC sheets $(200 \mathrm{~mm} \times 100 \mathrm{~mm}$ $\times 0.5 \mathrm{~mm})(\mathrm{SKK}$, Germany) were used as screen-printing substrates.

\subsection{Methods}

2.2.1. Oxidative Polymerisation of PEDOT Dispersion. The PEDOT aqueous dispersion was prepared by using an Ultrasonic Processor (model UP $400 \mathrm{~S}$ from Dr. Hielscher $\mathrm{GmbH}$ ) during the synthesis. As an example, ethylenedioxythiophene $(1 \mathrm{~mL}, 9.4 \mathrm{mmol})$ and $3.5 \mathrm{~g}$ of poly(styrene sulfonate, sodium salt) were dissolved in $100 \mathrm{~mL}$ of distilled water. To this mixture, an equimolar amount of ammonium peroxydisulphate $(6.58 \mathrm{~g}, 28.8 \mathrm{mmol})$ dissolved in $50 \mathrm{~mL}$ of water was added dropwise over a period of 4 minutes. After 1 hour of reaction under ultrasonic irradiation a dark blue PEDOT aqueous dispersion was obtained.

\subsubsection{Enzymatic Polymerisation of PEDOT Dispersion. The} enzymatic polymerisation of PEDOT was performed as described in [27]. In a typical polymerisation reaction, equimolar quantities (typically $0.055 \mathrm{M}$ ) of the template (PSS) and EDOT were dissolved into $20 \mathrm{~mL}$ of distilled water at room temperature. Next, the $\mathrm{pH}$ was adjusted to 2 with addition of $\mathrm{HCl}$. A monomer/template complex was formed spontaneously. $2 \mathrm{~mL}$ of HRP solution ( $3 \mathrm{mg}$ in $1 \mathrm{~mL}$ of distilled water) and an equimolar amount (typically $0.055 \mathrm{M}$ ) of hydrogen peroxide solution were added under magnetic 
stirring. Reaction was carried out at $4^{\circ} \mathrm{C}$ for 16 hours and a characteristic blue colour PEDOT solution was obtained.

2.2.3. Fabrication of PEDOT Modified Electrodes. Screenprinted electrodes were produced using a semi-automatic Thieme $1010 \mathrm{E}$, following a three-electrode configuration. The working electrode was a $4 \mathrm{~mm}$-diameter circle, the auxiliary electrode was a $16 \mathrm{~mm} \times 1.5 \mathrm{~mm}$ curved line and the $\mathrm{Ag} / \mathrm{AgCl}$ pseudo-reference electrode was a $5 \mathrm{~mm} \times 1.5 \mathrm{~mm}$ straight line. The PEDOT dispersion was directly screenprinted on the working electrode or deposited manually ( $3 \mu \mathrm{L}$ PEDOT solution per electrode using a micropipette). The electrodes were left to dry during $30 \mathrm{~min}$ at $60^{\circ} \mathrm{C}$.

2.2.4. Conductivity Measurements. The electrical conductivity of the mediator layers was measured on a thin film of the material printed on a glass substrate, using a standard fourprobe technique at room temperature (homemade instrument, CIDETEC, Spain). All the materials were measured at five different spots. Average data of the five measurements will be presented.

2.2.5. Immobilisation of Enzymes. The immobilisation of enzymes was performed by physical entrapment in a polyvinylalcohol-based photopolymer (PVA-SbQ), as described in previous works [13]. As a summary, PVA-SbQ polymer was mixed with B394-AChE solution in a ratio $1: 1(\mathrm{v} / \mathrm{v})$. The mixture was vortex-mixed and briefly centrifuged to eliminate the foam. A volume of $3 \mu \mathrm{L}$ was then spread onto the working electrode using a micropipette. The concentration of the initial enzymatic solution was adjusted in order to obtain a final enzyme loading of $1 \mathrm{mU} /$ biosensor.

The electrodes were exposed to a neon light for three 48 hours at $4^{\circ} \mathrm{C}$ to allow the photopolymerisation. After drying for next 48 hours at $4^{\circ} \mathrm{C}$, the biosensors were ready to use.

2.2.6. Cyclic Voltametry Measurements. Cyclic voltametry measurements were performed using an EPSILON Electrochemical analyser (BAS, Bioanalytical Systems, USA). The sensors-based on PEDOT were tested in a drop $(60 \mu \mathrm{L})$ of $0.1 \mathrm{M}$ phosphate buffer $\mathrm{pH}$ 7. The oxidation peak generated upon injection of thiocholine at a final concentration $25 \mathrm{mM}$ was determined by scanning the potential from -500 to $+500 \mathrm{mV}$ versus $\mathrm{Ag} / \mathrm{AgCl}$ at a scan rate of $5 \mathrm{mV} \cdot \mathrm{sec}^{-1}$.

2.2.7. Amperometric Measurements. Amperometric measurements were carried out with a BAS EPSILON (Bioanalytical Systems, USA). The measurements were performed in a drop $(60 \mu \mathrm{L})$ covering the three electrodes of the screenprinted sensor. The sensors were tested in $0.1 \mathrm{M}$ phosphate buffer $\mathrm{pH} 7$ at $100 \mathrm{mV}$ versus $\mathrm{Ag} / \mathrm{AgCl}$. The current intensity was recorded and, after current stabilisation, ATCh or thiocholine were injected to injected to a final concentration of $25 \mathrm{mM}$. ATCh was employed when the enzyme (AChE) was immobilised in the electrode, for measurement of pesticides. Thiocholine was used when there was no AChE in the sensor, especially for studying the mediating properties and stability of the mediator. Thiocholine has been obtained

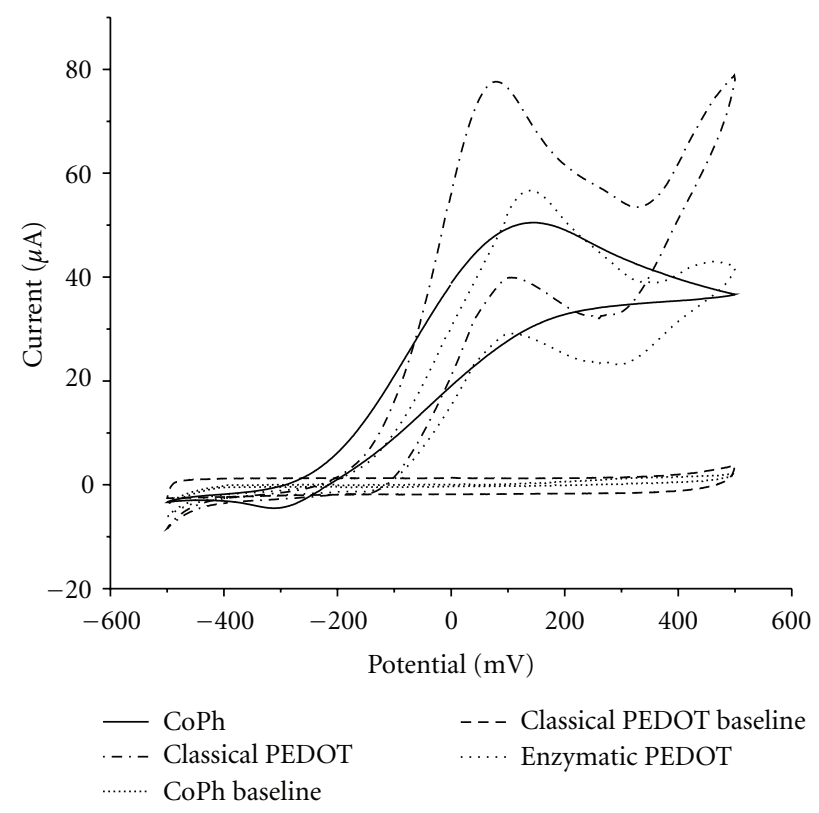

Figure 1: Cyclic voltamograms obtained in $0.1 \mathrm{M}$ phosphate buffer $\mathrm{pH} 7$ in absence or presence of thiocholine $25 \mathrm{mM}$. Comparison between electrodes modified with PEDOT or CoPC (scan rate: $\left.5 \mathrm{mV} \cdot \sec ^{-1}\right)$.

by direct hydrolysis of ATCh by AChE in a vial during 3 hours at room temperature. In both cases, either with ATCh or with thiocholine, the time necessary to reach the plateau was around $5 \mathrm{~min}$. The measured signal corresponded to the difference of current intensity between the baseline and the plateau. The electrode was washed with distilled water between measurements.

The pesticide detection was made in a three-step procedure as follows: first, the initial response of the electrode to $25 \mathrm{mM}$ ATCh was recorded three times, then the electrode was incubated in a solution containing a known concentration of insecticide, and finally the residual response of the electrode was recorded again. The percentage of inhibition was then correlated with the insecticide concentration.

\section{Results and Discussion}

The ability of PEDOT modified screen-printed electrodes to detect thiocholine was studied by cyclic voltametry, by scanning the potential between -500 to $500 \mathrm{mV}$ versus $\mathrm{Ag} / \mathrm{AgCl}$ pseudo reference electrode. Figure 1 shows the voltamograms obtained using screen-printed electrodes modified by PEDOT (classically or enzymatically synthesised) or CoPC, in presence of $25 \mathrm{mM}$ thiocholine. The sensors modified by CoPC presented an oxidation peak starting at $-200 \mathrm{mV}$ versus $\mathrm{Ag} / \mathrm{AgCl}$, characteristic of the mediated detection of thiocholine [34], while PEDOT modified electrodes, both classically and enzymatically synthesised showed an oxidation peak starting at $-100 \mathrm{mV}$ versus $\mathrm{Ag} / \mathrm{AgCl}$. As thiocholine oxidation is generally performed at a potential of $410 \mathrm{mV}$ versus $\mathrm{Ag} / \mathrm{AgCl}$ [35], the obtained results confirm that PEDOT could effectively be used as a new mediator in the amperometric detection of thiocholine. 
TABLE 1: Stability of the electrochemical response after being tested several times. Amperometric response to thiocholine $25 \mathrm{mM}$ at $100 \mathrm{mV}$. Comparison between oxidation currents (in $\mathrm{nA}$ ) from electrodes modified with CoPC classical and enzymatic PEDOT.

\begin{tabular}{lccc}
\hline & \multicolumn{3}{c}{ Currents of electrodes modified with [nA]: } \\
No & Classical & $\begin{array}{c}\text { Enzymatic } \\
\text { PEDOT }\end{array}$ & $\begin{array}{c}\text { Enzymatic } \\
\text { PEDOT + PVA layer }\end{array}$ \\
\hline 1 & PEDOT & 220 & 185 \\
2 & 310 & 205 & 196 \\
3 & 312 & 190 & 175 \\
4 & 318 & 87 & 207 \\
5 & 307 & 30 & 193 \\
6 & 302 & - & 211 \\
7 & 314 & - & 181 \\
8 & 321 & - & 173 \\
9 & 319 & - & 211 \\
10 & 330 & - & 184 \\
\hline
\end{tabular}

Once the ability of the PEDOT modified electrodes to mediate thiocholine oxidation was probed, the stability of these electrodes in successive thiocholine tests was checked. For this purpose, the electrodes response to thiocholine oxidation was measured during an amperometric process at $100 \mathrm{mV}$, as explained in Section 2.2.7. The intensity increase was recorded after 10 min of oxidation, in presence of thiocholine $25 \mathrm{mM}$, and the process was repeated several times with the same electrode. Values of subsequent testing are recorded in Table 1 . As it can be observed, the electrodes incorporating classically obtained PEDOT were proved to be stable for at least 12 consecutive tests, confirming an excellent stability of these PEDOTmodified electrodes. The electrodes modified by enzymatically synthesized PEDOT, on the other hand, were stable for only 3 consecutive drop depositions, showing a lower stability than the former. This decrease could be directly related with the exceptional solubility in water reached in this green synthesis process, provoking a release of the PEDOT layer from the electrode to the aqueous media and giving, as a consequence, a decrease in the signal of consecutive measurements. It is worth mentioning that the final biosensor device is composed by the deposition of an additional photocrosslinkable polymer layer (PVA-SbQ), containing the enzyme, that will additionally act as protector against leakage, as will be shown below. Therefore, the stability of the enzymatically synthesised PEDOT electrodes was also tested when the working electrode was covered by a layer of PVA-SbQ. No AChE was added at this stage, since thiocholine was previously produced out of the electrode. The stability of these sensors at successive amperometric measurements was dramatically improved using this protective layer (Table 1), the obtained sensors were now as resistant as their analogous sensors containing classical PEDOT, being stable for ten tests without significant lost in the response.
The following stage for the production of pesticide sensors was to immobilize AChE onto the surface of the PEDOT modified SPE. This way, AChE-based biosensors were obtained by entrapment of $1 \mathrm{mU}$ of AChE in a PVA-SbQ polymer as explained in Section 2.2.5. Then, the electrochemical characterisation of these biosensors was performed by measuring the oxidation current in presence of $25 \mathrm{mM}$ ATCh at $100 \mathrm{mV}$.

The poor stability observed for the enzymatic PEDOT layer in previous experiments disappeared when an additional photocrosslinkable polymer was deposited on it. This was already shown in previous experiments without AChE, where only PVA-SbQ was added (Table 1). Regarding the amperometric response, biosensors prepared using classically obtained PEDOT as mediator showed an average signal being 2 times higher than that one obtained with CoPC-modified electrodes, fact that implies that they will probably show higher sensitivity to the detection of pesticide. As for the biosensors containing enzymatically synthesised PEDOT, even though their amperometric response was not as pronounced as the one obtained with classical PEDOT-mediated electrodes, the values where still higher than those obtained for CoPC-modified ones. An average signal of $302 \pm 11 \mathrm{nA}$ (RSD = 3.6\%) was obtained for the biosensor-based on classical PEDOT (same biosensor, repeated measurements), while this signal was of $191 \pm 13 \mathrm{nA}$ (RSD $=7.1 \%)$ for the biosensor containing enzymatically synthesized PEDOT. As a comparison, conventional sensorsbased on CoPC displayed a signal of $158 \pm 10 \mathrm{nA}$ (RDS = $6.1 \%)$. In the opinion of the authors, the differences in the amperometric response between the electronic mediators are likely to be the result of the difference on the electrical conductivity of the working layer. The higher the electrical conductivity of the mediator, the greater is likely to be the amperometric response of the sensor. So, it seems logical to expect a higher amperometric response from a PEDOT-mediated biosensor than from CoPC-mediated one (a classical PEDOT having an intrinsic conductivity of $2 \mathrm{~S} / \mathrm{cm}$ gives a mediator layer showing $1271 \mathrm{~S} / \mathrm{cm}$ of conductivity once deposited onto the SPE; in contrast, the CoPC layer onto the SPE shows a conductivity of $671 \mathrm{~S} / \mathrm{cm}$ ). Similarly, a classical PEDOT-mediated biosensor shows higher amperometric response than that one containing enzymatic PEDOT (while the classical PEDOT has an intrinsic conductivity of $2 \mathrm{~S} / \mathrm{cm}$, the intrinsic conductivity observed for the enzymatic one is of $1.10^{-3} \mathrm{~S} / \mathrm{cm}$ ). As a result, the optimal inhibition rates that will be reached using these two new types of electrodes are related to their high amperometric responses.

The inhibition effect of CPO pesticide on AChE-based biosensor was studied after the incubation of the electrode for $10 \mathrm{~min}$ in a solution containing the pesticide. This incubation time was optimised in a previous work by the same authors, and concluded to be the time required for a total inhibition at different pesticide concentrations [7, 36, 37]. As previously suggested, the high average signal obtained with PEDOT modified electrodes gave a first idea about the promising sensibility of these biosensors. These first impressions were verified with inhibition measurements: 


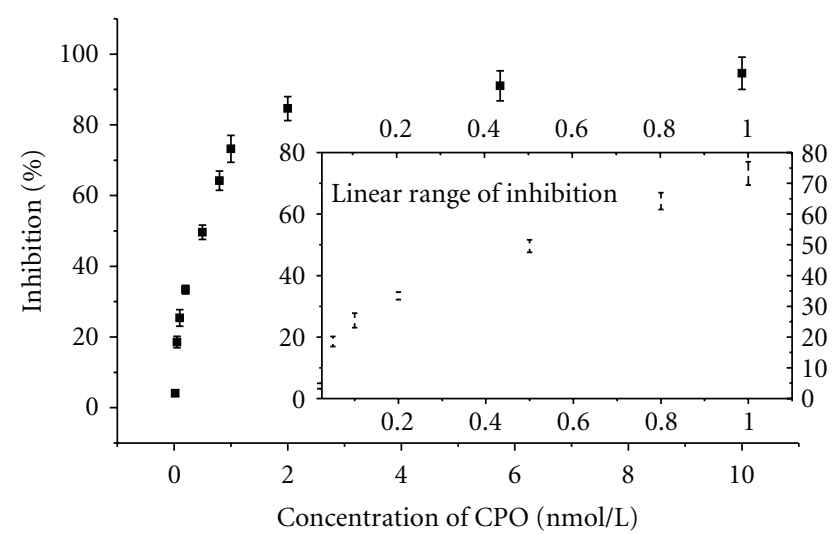

FIGURE 2: Inhibition ratios obtained with various concentrations of $\mathrm{CPO}$ for a biosensor based on a classical PEDOT as mediator.

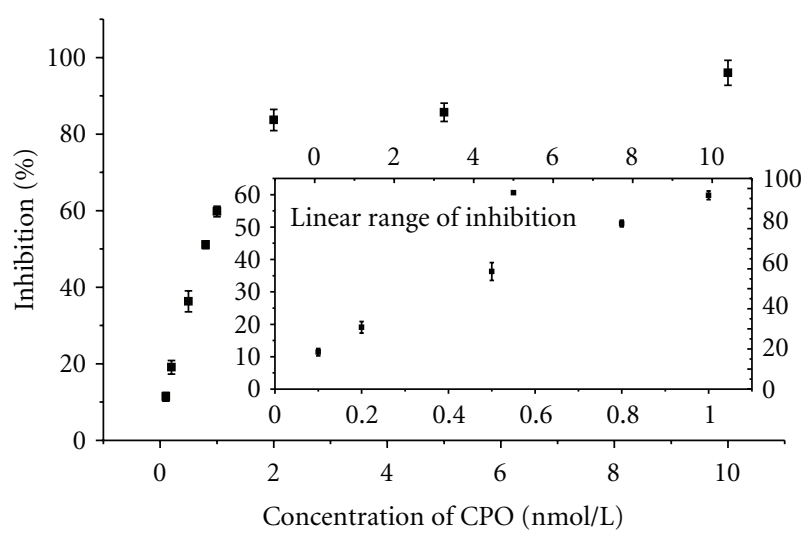

FIGURE 3: Inhibition ratios obtained with various concentrations of $\mathrm{CPO}$ for a biosensor based on an enzymatic PEDOT as mediator.

while the limits of detection (LOD) were calculated with basis of $10 \%$ enzyme inhibition with other classical mediatorsbased biosensors like CoPC or TCNQ [7, 36, 37], the sensors modified by PEDOT allowed to reach LOD on the concentration level of $10^{-11} \mathrm{M}$ (calculated using definition of LOD as 3 times standard deviation of the blank solution) and accurate measurements of inhibition with $\mathrm{LOQ}=10^{-10} \mathrm{M}$. Figures 2 and 3 show the calibration curve for the inhibition of the PEDOT-mediated biosensors with $\mathrm{CPO}$ pesticide concentrations, from 0.02 to $10 \mathrm{nmol} / \mathrm{L}$ of $\mathrm{CPO}$ when a classical PEDOT is used (Figure 2) and from 0.1 to $10 \mathrm{nmol} / \mathrm{L}$ of $\mathrm{CPO}$ when an enzymatic PEDOT is used (Figure 3 ).

As a final experiment, and taking into account that this PEDOT dispersion provides new opportunities to a high-scale massproduction, reproducibility measurements were carried out taking as reference a specific concentration of CPO pesticide $\left(5 \times 10^{-10} \mathrm{~mol} / \mathrm{L}\right)$. In this case, the reproducibility of 10 electrodes was studied, all of them fabricated in the same production series, using a classically synthesised PEDOT. Figure 4 shows the excellent reproducibility obtained with an average inhibition rate of $48.6 \pm 1.5 \%$ with $\mathrm{RDS}=3.1 \%$.

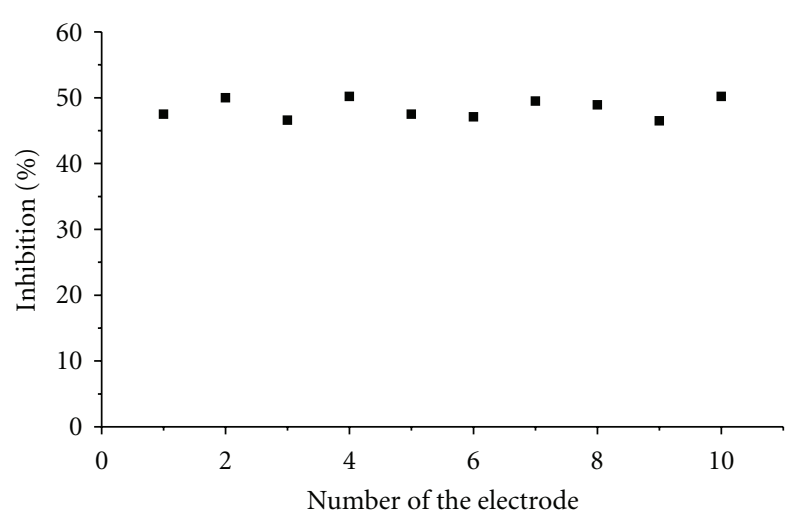

FIGURE 4: Reproducibility of the inhibition. Average inhibition rate for the classical PEDOT modified biosensors.

\section{Conclusions}

Two chemically synthesised poly(3,4-ethylenedioxythiophene) (PEDOT) were studied as electrochemical mediators in acetylcholinesterase (AChE)-based screen-printed biosensors, each one fabricated using a different synthesis strategy. In both cases, the use of a water-soluble conducting polymer as mediator in screen-printed biosensors enables its deposition by printing, making feasible a high-scale mass production of such biosensor devices. Both PEDOTmediated (AChE)-based screen-printed biosensors show higher average signal in the amperometric oxidation of thiocholine at $100 \mathrm{mV}$ than classically used CoPC-modified electrodes; an average signal of $302 \pm 11 \mathrm{nA}$ with RDS $=3.6 \%$ was obtained for a classical PEDOT modified biosensor and an average signal of $191 \pm 13 \mathrm{nA}$ with RDS $=7.1 \%$ for an enzymatic PEDOT modified one. As a consequence of the high thiocholine oxidation currents shown by these mediated biosensors, the detection of the pesticide $\mathrm{CPO}$ reached a LOD on the concentration level of $10^{-11} \mathrm{M}$ and accurate measurements of inhibition rates with $\mathrm{LOQ}=10^{-10} \mathrm{M}$.

Both polymers showed to be of extreme interest opening new opportunities for a high-scale mass production of biosensor devices.

\section{References}

[1] S. Andreescu, L. Barthelmebs, and J. L. Marty, "Immobilization of acetylcholinesterase on screen-printed electrodes: comparative study between three immobilization methods and applications to the detection of organophosphorus insecticides," Analytica Chimica Acta, vol. 464, no. 2, pp. 171-180, 2002.

[2] F. J. Rawson, W. M. Purcell, J. Xu et al., "A microband lactate biosensor fabricated using a water-based screen-printed carbon ink," Talanta, vol. 77, no. 3, pp. 1149-1154, 2009.

[3] R. M. Pemberton, R. Pittson, N. Biddle, and J. P. Hart, "Fabrication of microband glucose biosensors using a screenprinting water-based carbon ink and their application in serum analysis," Biosensors and Bioelectronics, vol. 24, no. 5, pp. 1246-1252, 2009. 
[4] E. Crouch, D. C. Cowell, S. Hoskins, R. W. Pittson, and J. P. Hart, "Amperometric, screen-printed, glucose biosensor for analysis of human plasma samples using a biocomposite water-based carbon ink incorporating glucose oxidase," Analytical Biochemistry, vol. 347, no. 1, pp. 17-23, 2005.

[5] E. Crouch, D. C. Cowell, S. Hoskins, R. W. Pittson, and J. P. Hart, "A novel, disposable, screen-printed amperometric biosensor for glucose in serum fabricated using a water-based carbon ink," Biosensors and Bioelectronics, vol. 21, no. 5, pp. 712-718, 2005.

[6] A. A. Ciucu, C. Negulescu, and R. P. Baldwin, "Detection of pesticides using an amperometric biosensor based on ferophthalocyanine chemically modified carbon paste electrode and immobilized bienzymatic system," Biosensors and Bioelectronics, vol. 18, no. 2-3, pp. 303-310, 2003.

[7] G. S. Nunes, D. Barceló, B. S. Grabaric, J. M. Díaz-Cruz, and M. L. Ribeiro, "Evaluation of a highly sensitive amperometric biosensor with low cholinesterase charge immobilized on a chemically modified carbon paste electrode for trace determination of carbamates in fruit, vegetable and water samples," Analytica Chimica Acta, vol. 399, no. 1-2, pp. 37-49, 1999.

[8] G. S. Nunes, P. Skládal, H. Yamanaka, and D. Barceló, "Determination of carbamate residues in crop samples by cholinesterase- based biosensors and chromatographic techniques," Analytica Chimica Acta, vol. 362, no. 1, pp. 59-68, 1998.

[9] P. Skládal and M. Maschini, "Sensitive detection of pesticides using amperometric sensors based on cobalt phthalocyanine-modified composite electrodes and immobilized cholinesterases," Biosensors and Bioelectronics, vol. 7, no. 5, pp. 335-343, 1992.

[10] P. Skladal, "Detection of organophosphate and carbamate pesticides using disposable biosensors based on chemically modified electrodes and immobilized cholinesterase," Analytica Chimica Acta, vol. 269, no. 2, pp. 281-287, 1992.

[11] H. Schulze, S. Vorlová, F. Villatte, T. T. Bachmann, and R. D. Schmid, "Design of acetylcholinesterases for biosensor applications," Biosensors and Bioelectronics, vol. 18, no. 2-3, pp. 201-209, 2003.

[12] C. Bonnet, S. Andreescu, and J. L. Marty, "Adsorption: an easy and efficient immobilisation of acetylcholinesterase on screenprinted electrodes," Analytica Chimica Acta, vol. 481, no. 2, pp. 209-211, 2003.

[13] S. Andreescu, L. Barthelmebs, and J. L. Marty, "Immobilization of acetylcholinesterase on screen-printed electrodes: comparative study between three immobilization methods and applications to the detection of organophosphorus insecticides," Analytica Chimica Acta, vol. 464, no. 2, pp. 171-180, 2002.

[14] T. T. Bachmann, B. Leca, F. Vilatte, J. L. Marty, D. Fournier, and R. D. Schmid, "Improved multianalyte detection of organophosphates and carbamates with disposable multielectrode biosensors using recombinant mutants of Drosophila acetylcholinesterase and artificial neural networks," Biosensors and Bioelectronics, vol. 15, no. 3-4, pp. 193-201, 2000.

[15] D. Martorell, F. Céspedes, E. Martínez-Fàbregas, and S. Alegret, "Determination of organophosphorus and carbamate pesticides using a biosensor based on a polishable, 7,7,8,8-tetracyanoquinodimethane-modified, graphite-epoxy biocomposite," Analytica Chimica Acta, vol. 337, no. 3, pp. 305-313, 1997.

[16] R. Nagarajan, S. Tripathy, J. Kumar, F. F. Bruno, and L. Samuelson, "Manipulating DNA conformation using inter- twined conducting polymer chains," Macromolecules, vol. 33, pp. 9542-9547, 2000.

[17] W. Liu, J. Kumar, S. Tripathy, K. J. Senecal, and L. Samuelson, "Enzymatically synthesized conducting polyaniline," Journal of the American Chemical Society, vol. 121, no. 1, pp. 71-78, 1999.

[18] S. Cosnier, C. Gondran, and A. Senillou, "Functionalized polypyrroles: a sophisticated glue for the immobilization and electrical wiring of enzymes," Synthetic Metals, vol. 102, no. 1-3, pp. 1366-1369, 1999.

[19] S. Cosnier, M. Stoytcheva, A. Senillou, H. Perrot, R. P. M. Furriel, and F. A. Leone, "A biotinylated conducting polypyrrole for the spatially controlled construction of an amperometric biosensor," Analytical Chemistry, vol. 71, no. 17, pp. 3692-3697, 1999.

[20] P. He, J. Ye, Y. Fang, J. Anzai, and T. Osa, "Self-assembled biotinylated disulfide derivative monolayer on gold electrode for immobilizing enzymes," Talanta, vol. 44, no. 5, pp. 885890, 1997.

[21] J. Stejskal, "Colloidal dispersions of conducting polymers," Journal of Polymer Materials, vol. 18, no. 3, pp. 225-258, 2001.

[22] M. Thiyagarajan, L. A. Samuelson, J. Kumar, and A. L. Cholli, "Helical conformational specificity of enzymatically synthesized water-soluble conducting polyaniline nanocomposites," Journal of the American Chemical Society, vol. 125, no. 38, pp. 11502-11503, 2003.

[23] X. Wang, H. Schreuder-Gibson, M. Downey, S. Tripathy, and L. Samuelson, "Conductive fibers from enzymatically synthesized polyaniline," Synthetic Metals, vol. 107, no. 2, pp. 117-121, 1999.

[24] W. Liu, A. L. Cholli, R. Nagarajan et al., "The role of template in the enzymatic synthesis of conducting polyaniline," Journal of the American Chemical Society, vol. 121, no. 49, pp. 11345$11355,1999$.

[25] W. Liu, A. L. Cholli, J. Kumar, S. Tripathy, and L. Samuelson, "Mechanistic study of the peroxidase-catalyzed polymerization of sulfonated phenol," Macromolecules, vol. 34, no. 11, pp. 3522-3526, 2001.

[26] V. Rumbau, R. Marcilla, E. Ochoteco, J. A. Pomposo, and D. Mecerreyes, "Ionic liquid immobilized enzyme for biocatalytic synthesis of conducting polyaniline," Macromolecules, vol. 39, no. 25, pp. 8547-8549, 2006.

[27] V. Rumbau, J. A. Pomposo, A. Eleta et al., "First enzymatic synthesis of water-soluble conducting poly(3,4ethylenedioxythiophene)," Biomacromolecules, vol. 8, no. 2, pp. 315-317, 2007.

[28] T. Sikora, R. Marcilla, D. Mecerreyes, J. Rodriguez, J. A. Pomposo, and E. Ochoteco, "Enzymatic synthesis of watersoluble conducting poly(3,4- ethylenedioxythiophene): a simple enzyme immobilization strategy for recycling and reusing," Journal of Polymer Science A, vol. 47, no. 1, pp. 306-309, 2009.

[29] R. Cruz-Silva, J. Romero-García, J. L. Angulo-Sánchez et al., "Template-free enzymatic synthesis of electrically conducting polyaniline using soybean peroxidase," European Polymer Journal, vol. 41, no. 5, pp. 1129-1135, 2005.

[30] C. H. Lim and Y. J. Yoo, "Synthesis of ortho-directed polyaniline using horseradish peroxidase," Process Biochemistry, vol. 36, no. 3, pp. 233-241, 2000.

[31] M. Thiyagarajan, L. A. Samuelson, J. Kumar, and A. L. Cholli, "Helical conformational specificity of enzymatically synthesized water-soluble conducting polyaniline nanocomposites," Journal of the American Chemical Society, vol. 125, no. 38, pp. 11502-11503, 2003. 
[32] P. Xu, A. Singh, and D. L. Kaplan, "Enzymatic catalysis in the synthesis of polyanilines and derivatives of polyanilines," Advances in Polymer Science, vol. 194, no. 1, pp. 69-94, 2006.

[33] G. Istamboulie, T. Sikora, E. Jubete, E. Ochoteco, J. L. Marty, and T. Noguer, "Screen-printed poly(3,4-ethylenedioxythiophene) (PEDOT): a new electrochemical mediator for acetylcholinesterase-based biosensors," Talanta, vol. 82, pp. 957-961, 2010.

[34] G. Istamboulie, D. Fournier, J. L. Marty, and T. Noguer, "Phosphotriesterase: a complementary tool for the selective detection of two organophosphate insecticides: chlorpyrifos and chlorfenvinfos," Talanta, vol. 77, no. 5, pp. 1627-1631, 2009.

[35] P. Skadal, "Determination of organophosphate and carbamate pesticides using a cobalt phthalocyanine-modified carbon paste electrode and a cholinesterase enzyme membrane," Analytica Chimica Acta, vol. 252, no. 1-2, pp. 11-15, 1991.

[36] G. S. Nunes, T. Montesinos, P. B. O. Marques, D. Fournier, and J. L. Marty, "Acetylcholine enzyme sensor for determining methamidophos insecticide-evaluation of some genetically modified acetylcholinesterases from Drosophila melanogaster," Analytica Chimica Acta, vol. 434, no. 1, pp. 1-8, 2001.

[37] P. Skládal, G. S. Nunes, H. Yamanaka, and M. L. Ribeiro, "Detection of carbamate pesticides in vegetable samples using cholinesterase-based biosensors," Electroanalysis, vol. 9, no. 14, pp. 1083-1087, 1997. 

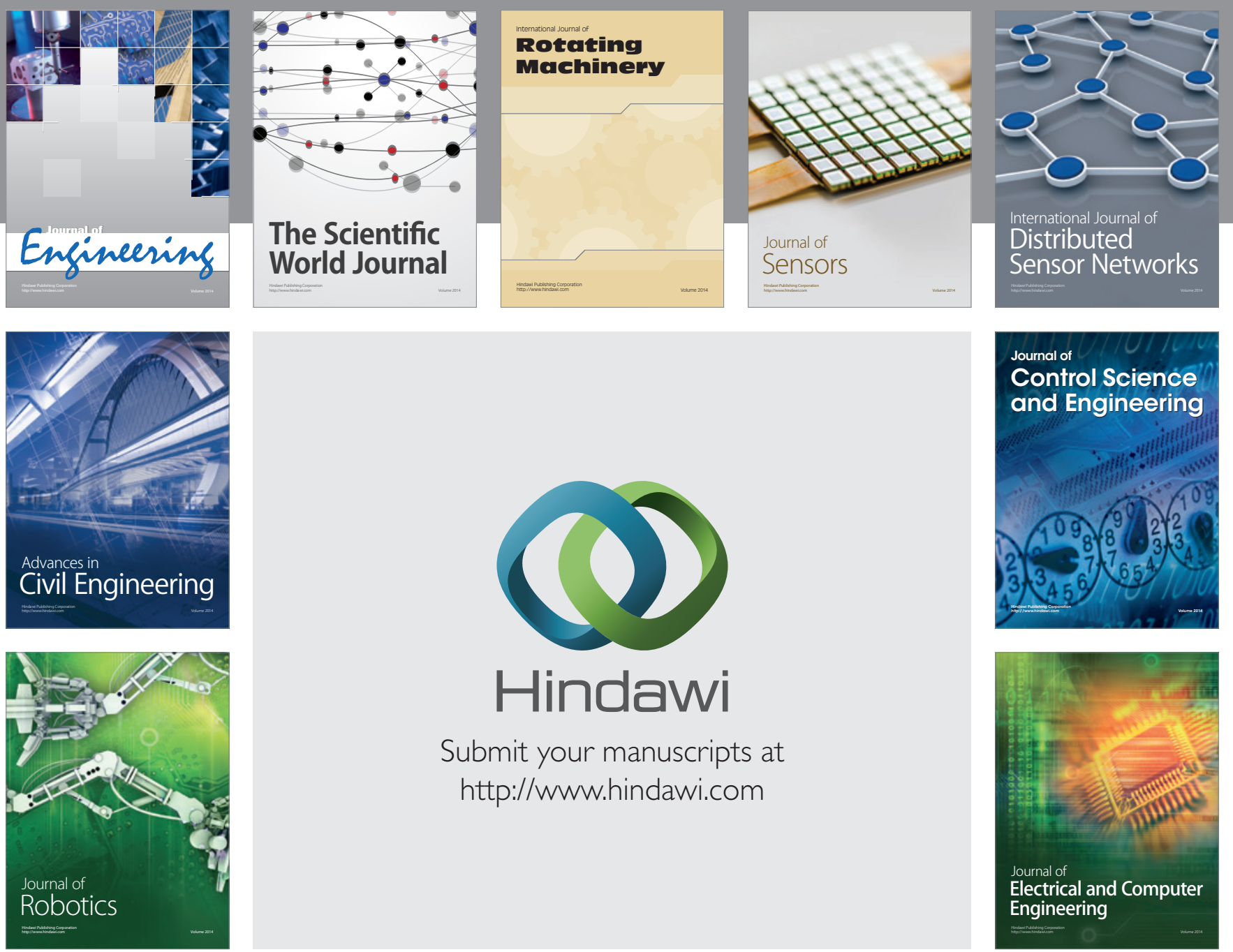

Submit your manuscripts at

http://www.hindawi.com
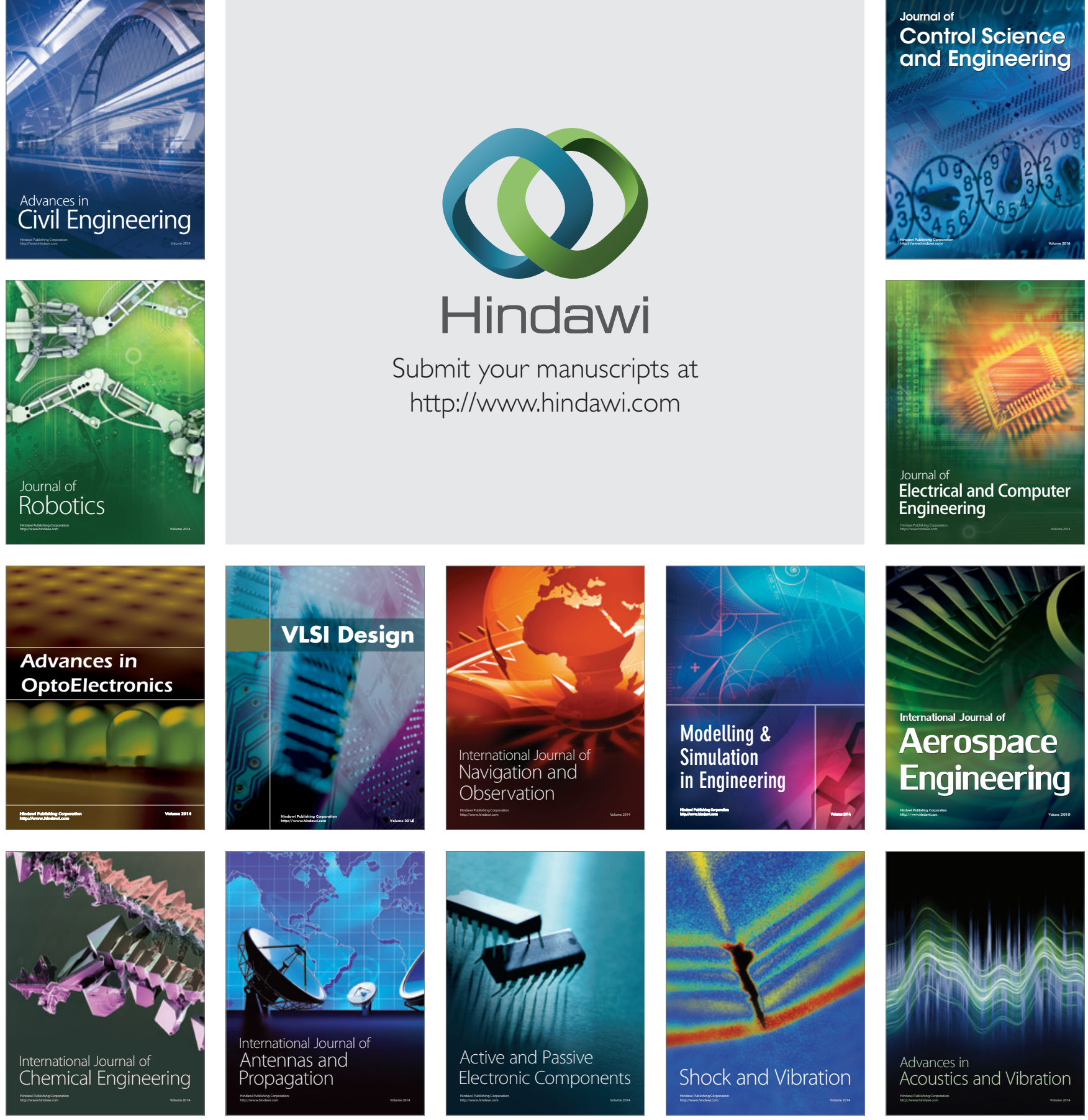